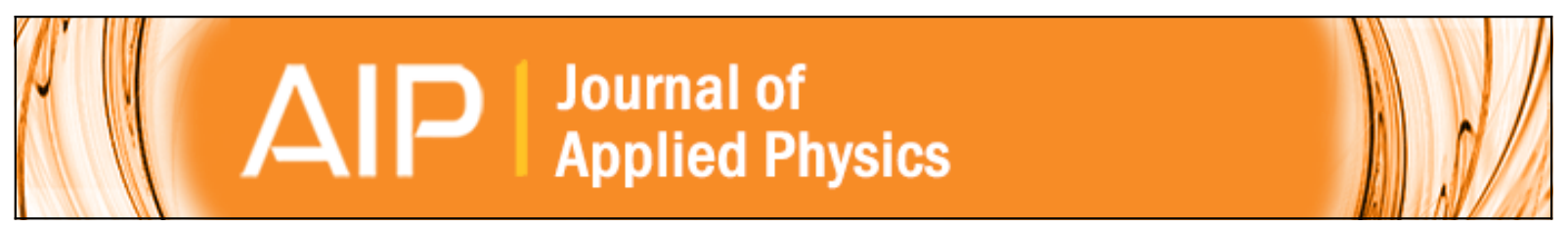

\title{
Perpendicular magnetic anisotropy of ColPt bilayers on ALD HfO2
}

Bart F. Vermeulen, Jackson Wu, Johan Swerts, Sebastien Couet, Dimitri Linten, Iuliana P Radu, Kristiaan Temst

, Geert Rampelberg, Christophe Detavernier, Guido Groeseneken, and Koen Martens

Citation: Journal of Applied Physics 120, 163903 (2016); doi: 10.1063/1.4966121

View online: http://dx.doi.org/10.1063/1.4966121

View Table of Contents: http://scitation.aip.org/content/aip/journal/jap/120/16?ver=pdfcov

Published by the AIP Publishing

\section{Articles you may be interested in}

Effect of substrate temperature on the magnetic properties of epitaxial sputter-grown Co/Pt

Appl. Phys. Lett. 103, 262401 (2013); 10.1063/1.4856395

Effects of Pt capping layer on perpendicular magnet anisotropy in pseudo-spin valves of

$\mathrm{Ta} / \mathrm{CoFeB} / \mathrm{MgO} / \mathrm{CoFeB} / \mathrm{Pt}$ magnetic-tunneling junctions

Appl. Phys. Lett. 102, 212409 (2013); 10.1063/1.4808084

Co/Pt multilayer-based magnetic tunnel junctions with perpendicular magnetic anisotropy

J. Appl. Phys. 111, $07 C 703$ (2012); 10.1063/1.3670972

Effect of annealing on the magnetic tunnel junction with Co/Pt perpendicular anisotropy ferromagnetic multilayers J. Appl. Phys. 107, 09 C711 (2010); 10.1063/1.3358249

Interdiffusion in epitaxial Co/Pt multilayers

J. Appl. Phys. 81, 637 (1997); 10.1063/1.364221

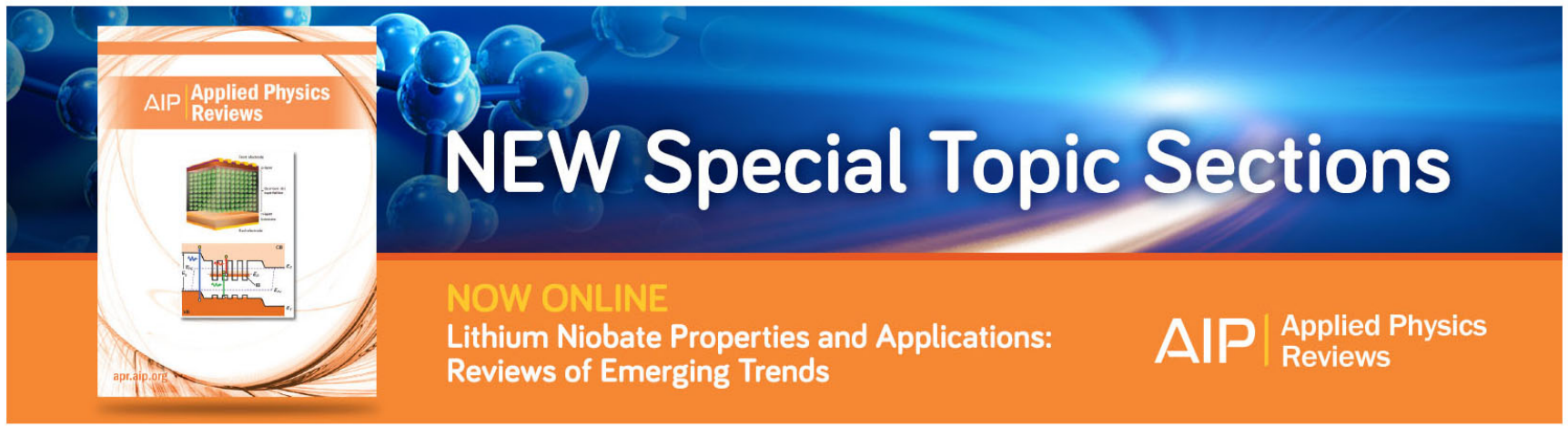




\title{
Perpendicular magnetic anisotropy of ColPt bilayers on ALD $\mathrm{HfO}_{2}$
}

\author{
Bart F. Vermeulen, ${ }^{1,2, a)}$ Jackson $\mathrm{Wu},{ }^{2}$ Johan Swerts, ${ }^{2}$ Sebastien Couet, ${ }^{2}$ Dimitri Linten, ${ }^{2}$ \\ Iuliana P Radu, ${ }^{2}$ Kristiaan Temst, ${ }^{1}$ Geert Rampelberg, ${ }^{3}$ Christophe Detavernier, ${ }^{3}$ \\ Guido Groeseneken, ${ }^{2,4}$ and Koen Martens ${ }^{2,1}$ \\ ${ }^{1}$ Department of Physics and Astronomy, KU Leuven, Leuven, Belgium \\ ${ }^{2}$ IMEC, Kapeldreef 75, Leuven, Belgium \\ ${ }^{3}$ Solid State Physics, Ghent University, Ghent, Belgium \\ ${ }^{4}$ Department of Electrical Engineering, KU Leuven, Leuven, Belgium
}

(Received 4 August 2016; accepted 12 October 2016; published online 27 October 2016)

\begin{abstract}
Perpendicular Magnetic Anisotropy (PMA) is a key requirement for state of the art Magnetic Random Access Memories (MRAM). Currently, PMA has been widely reported in standard Magnetic Tunnel Junction material stacks using $\mathrm{MgO}$ as a dielectric. In this contribution, we present the first report of PMA at the interface with a high- $\kappa$ dielectric grown by Atomic Layer Deposition, $\mathrm{HfO}_{2}$. The PMA appears after annealing a $\mathrm{HfO}_{2} \backslash \mathrm{Co} \backslash \mathrm{Pt} \mathrm{Ru}$ stack in $\mathrm{N}_{2}$ with the $\mathrm{K}_{\text {eff }}$ of $0.25 \mathrm{~mJ} / \mathrm{m}^{2}$ as determined by Vibrating Sample Magnetometry. X-Ray Diffraction and Transmission Electron Microscopy show that the appearance of PMA coincides with interdiffusion and the epitaxial ordering of the Co\Pt bilayer. High- $\kappa$ dielectrics are especially interesting for Voltage Control of Magnetic Anisotropy applications and are of potential interest for low-power MRAM and spintronics technologies. Published by AIP Publishing. [http://dx.doi.org/10.1063/1.4966121]
\end{abstract}

\section{INTRODUCTION}

Perpendicular Magnetic Anisotropy (PMA) has been widely reported in several stacks of metallic multilayers. ${ }^{1}$ Co\Pt bilayers and superlattices deposited on crystalline templates show PMA as deposited. ${ }^{2}$ The saturation magnetization of Co\Pt multilayers on crystalline templates increases (from the value of bulk Co, $1446 \mathrm{kA} / \mathrm{m}$ ) with decreasing Co thicknesses to values ranging between 1600 and $1850 \mathrm{kA} / \mathrm{m}$ for films with the Co thickness of $5 \AA$, ${ }^{3,4}$ depending on the annealing conditions and the template material. The effective anisotropy energy for such films is reportedly between 0.3 and $0.32 \mathrm{~mJ} / \mathrm{m}^{2} /$ interface ${ }^{3,5}$ in the PMA regime. These values are reported for CoVt multilayers with the strong (111) texture and are dependent on the Co and Pt thicknesses, ${ }^{3,5}$ the sharpness of the Co\t interface, ${ }^{6,7}$ the annealing conditions, and the crystallinity of the template. ${ }^{4}$

$\mathrm{HfO}_{2}$ on the other hand is amorphous. Co\t bilayer structures have already been studied on other amorphous oxides like $\mathrm{SiO}_{2}$ by Nistor et al. ${ }^{8}$ They report that an annealing temperature from $250{ }^{\circ} \mathrm{C}$ to $400^{\circ} \mathrm{C}$ can modify the Magnetic Anisotropy (MA) of a thin Co film from in-plane (IP) to perpendicular (PMA). The oxygen atoms at the interface between Co and the oxide may also contribute to the appearance of PMA, through hybridization of the Co $3 \mathrm{~d}_{z}$ orbitals with the oxide $2 \mathrm{p}_{x y(y z)}$ orbitals. ${ }^{9,10}$

The anisotropy energy of Co\Pt layers is constant in function of the annealing temperature, until some critical temperature $\mathrm{T}_{c}$ is reached. Above this $\mathrm{T}_{c}$, the anisotropy energy will decrease in function of the annealing temperature. ${ }^{11-13}$ Improving the crystallinity of the seed layer can significantly increase the magnetic anisotropy energy $\mathrm{K}_{\text {eff }}$ at a given annealing temperature ${ }^{11}$ as well as the critical temperature. ${ }^{4,11}$

a)bart.vermeulen@imec.be
$K_{\text {eff }}$ is an important figure of merit for Voltage Control of Magnetic Anisotropy (VCMA) in ferromagnetic materials, which has attracted increasing attention since the first experimental evidence of the phenomenon in liquid electrolytes by Weisheit et al. ${ }^{14}$ In VCMA, a voltage is applied across a dielectric in contact with a ferromagnetic material, resulting in an electric field at the interface between the ferromagnet and the dielectric. The charging of the interface leads to a modification of the magnetic anisotropy. The VCMA effect was also found in solid state systems ${ }^{15-17}$ and is of significant importance for the electronics industry, as it would enable key technological advances in Magnetic Random Access Memories (MRAM) and spintronics. Until now, research focused primarily on typical perpendicular Magnetic Tunnel Junction (pMTJ) materials, i.e., $\mathrm{MgO}$ as a dielectric combined with $\mathrm{Co},{ }^{18} \mathrm{CoFe},{ }^{17,19,20} \mathrm{FeB},{ }^{21}$ or $\mathrm{CoFeB},{ }^{22-25}$ as well as $\mathrm{Fe},{ }^{16,26} \mathrm{~L}_{0} \mathrm{FePt}^{27}$ and $\mathrm{L} 1_{0} \mathrm{FePd}^{28}$

This work focuses on obtaining PMA onto $\mathrm{HfO}_{2}$ and provides an explanation for the behavior of the Co ferromagnetic layer in the function of the annealing temperature. Amorphous atomic layer deposition (ALD) $\mathrm{HfO}_{2}$ was chosen to avoid charge trapping in the oxide and lattice mismatch issues between $\mathrm{HfO}_{2}$ and Co. The thermal treatment to which the materials stacks are exposed promotes the interdiffusion of atoms between layers as well as the epitaxial relation between $\mathrm{Co}$ and $\mathrm{Pt}$ and can induce interfacial alloying, together leading to the appearance of PMA.

\section{EXPERIMENTAL}

Samples are fabricated on a $\mathrm{Si}$ wafer after an $\mathrm{O}_{3}$ based clean, leaving $1 \mathrm{~nm}$ of chemical $\mathrm{SiO}_{2}$. A forming gas anneal at $420^{\circ} \mathrm{C}$ for $20 \mathrm{~min}$ is applied to passivate dangling bonds, after which $2.5 \mathrm{~nm}$ of amorphous ${ }^{29}$ wet ALD $\mathrm{HfO}_{2}$ is deposited at $300^{\circ} \mathrm{C}$ in an ASM Polygon 8300 reactor. The samples 
are transferred to a Canon Anelva PVD reactor, the $\mathrm{HfO}_{2}$ layer is degassed in UHV at $350^{\circ} \mathrm{C}$ for $5 \mathrm{~min}$, and a $\mathrm{Co}$ wedge with thickness ranging from 0.6 to $2.4 \mathrm{~nm}$ is sputterdeposited. Finally, $4 \mathrm{~nm}$ of Pt followed by $5 \mathrm{~nm}$ of $\mathrm{Ru}$ is deposited. Samples are annealed at temperatures between 250 and $350^{\circ} \mathrm{C}$ at atmospheric pressure for $10 \mathrm{~min}$ in $\mathrm{N}_{2}$ ambient.

X-Ray Diffraction (XRD) and Reflection (XRR) measurements are performed in a Panalytical X'pert X-Ray Diffractometer, with Bragg-Brentano configuration and $\mathrm{Cu}-$ $\mathrm{K} \alpha$ wavelength. In-situ XRD is performed in a Bruker D8 Discover equipped with a home-built annealing chamber at a constant heating rate of $0.5^{\circ} \mathrm{C} / \mathrm{s}$. The XRD pattern is recorded in a fixed $20^{\circ} 2 \theta$ window. The XRR characteristics are fitted using the Bruker Leptos software taking into account the following stack properties: densities, thicknesses, and interfacial intermixing as characterized by the standard deviation $\sigma$ of a Gaussian density intermixing profile at the $\mathrm{Co} / \mathrm{Pt}$ interface.

Transmission Electron Microscopy specimens are prepared using conventional ion milling and observed in a FEI Titan at $300 \mathrm{kV}$.

Rutherford Backscattering Spectra were obtained using a $\mathrm{He}^{+}$beam with an energy of $1.523 \mathrm{MeV}$, at a scattering angle of $170^{\circ}$ and a sample tilt angle of $11^{\circ}$.

Atomic Force Microscopy (AFM) measurements are collected with a Bruker edge AFM on a $5 \times 5 \mu \mathrm{m}^{2}$ area. They are analyzed using the Nanoscope Analysis software.

\section{RESULTS AND DISCUSSION}

\section{A. Structural characterization}

The XRD analysis is used for thin film phase identification. The blue vertical lines in Figure 1(a) show the positions of the XRD peak attributed to the thin films. The three visible peaks at $2 \theta$ angles of $39.9^{\circ}, 41.1^{\circ}$, and $42.4^{\circ}$ are analyzed by fitting a set of three Gaussian peaks with the least squares method, which allows to determine peak position with an accuracy of $0.1^{\circ}$. First, the peak at $39.9^{\circ}$ can be attributed to the fcc (111) platinum layer. ${ }^{11}$ The feature at $42.4^{\circ}$ can be ascribed to hcp (002) ruthenium. ${ }^{30}$ The peak at $41.1^{\circ}$ can be attributed to both the fcc (111) equivalent plane of a CoPt $\mathrm{L} 1_{0}$ alloy $^{31-33}$ or an fcc (111) CoPt alloy originating from

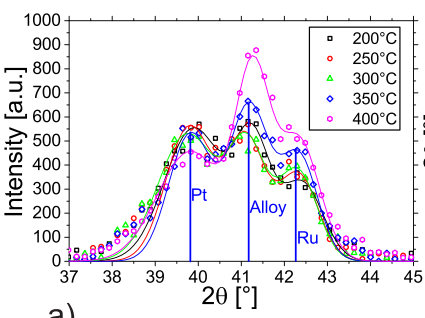

a)

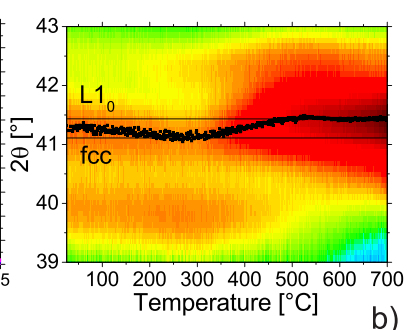

b)
FIG. 1. (a) The temperature dependence of the XRD diffractogram for in situ annealed $\mathrm{HfO}_{2} \backslash \mathrm{Co} \backslash \mathrm{PtRu}$ samples in the temperature range of $200^{\circ} \mathrm{C}-400^{\circ} \mathrm{C}$. The symbols show the raw data, and the lines show the Gaussian fits. The vertical blue lines mark the characteristic Pt, CoPt alloy, and Ru peak position after annealing at $350^{\circ} \mathrm{C}$ for $10 \mathrm{~min}$. (b) Two dimensional plot of the XRD amplitude as function of the sample temperature and the $2 \theta$ angle. The horizontal lines indicate the angles of the fcc alloy $\left(41.1^{\circ}\right)$ and $\mathrm{L}_{0}\left(41.4^{\circ}\right)$ peaks, respectively. The black dots indicate the angle of the central peak as a function of the temperature. interdiffusion. ${ }^{33}$ Bulk Co tends to crystallize in hep. The expected hcp (002) peak of Co $\left(44.5^{\circ}\right)$ is absent in the diffractogram (see Figure 1(a)), allowing to rule out this phase. The diffractogram shows a relaxed Pt peak as mentioned earlier $\left(39.9^{\circ}\right)$. Hence, the commensurate Pt contribution to the alloy peak is approximated by half of the Pt thickness, which allows the matching of the measured peak at $41.1^{\circ}$ with the following formula:

$$
\frac{2 \sin \theta}{\lambda}=\frac{1}{\|d\|} \quad \text { with } \quad\|d\|=\frac{n_{C o} d_{C o}+n_{P t} d_{P t}}{n_{C o}+n_{P t}},
$$

where $\lambda$ is the $\mathrm{Cu}-\mathrm{K} \alpha$ wavelength $(0.154056 \mathrm{~nm})$ and $\|d\|$ is the average interplanar distance, weighted with the layer thicknesses $\left(n_{C o}=6\right.$ and $n_{P t}=10$ are the as-deposited amount of monolayers and $d_{C o, P t}$ the absolute interplanar distances).

Figure 1(b) shows the evolution of the XRD characteristic in function of the in situ annealing temperature. At room temperature, the Pt and the alloy peak are already visible, showing that the as deposited samples have weak fcc (111) texture. The central peak angle shifts downwards towards the calculated fcc alloy angle $\left(41.1^{\circ}\right)$ as $\mathrm{T}_{\text {anneal }}$ increases to $300^{\circ} \mathrm{C}$. With the coefficient of thermal expansion of about $13 \mu \mathrm{K}^{-1}$ for $\mathrm{Co}$ and 8.8 for Pt, the central peak is expected to shift downward slightly less than $0.2^{\circ}$ between RT and $300^{\circ} \mathrm{C}$. Above $300^{\circ} \mathrm{C}$, the central peak moves upwards towards the $\mathrm{L} 1_{0}$ peak for higher temperatures, which is indicative of an ordering of the interfacial CoPt alloy into the $\mathrm{L}_{0}$ phase. Complete ordering of a CoPt alloy from fcc into an $\mathrm{L}_{0}$ tetragonal structure is reported to require annealing temperatures of typically $600^{\circ} \mathrm{C} \cdot{ }^{32,34,35}$ As the annealing temperature increases from $300^{\circ} \mathrm{C}$ to $500^{\circ} \mathrm{C}$ (see Figure $1(\mathrm{~b})$ ), the Pt peak gradually disappears. At the same time, the central peak angle shifts to a $2 \theta$ value of $41.4^{\circ}$, which corresponds to the (101) peak of tetragonally ordered $\mathrm{L}_{0} \mathrm{CoPt}^{32}$ (the $\mathrm{L} 1_{0}$ equivalent of the fcc (111) peak). As the transition to the $\mathrm{L} 1_{0}$ phase happens well above $350^{\circ} \mathrm{C}$, it can be ruled out as the origin of the central peak present at $41.1^{\circ}$ for anneals at $200-350^{\circ} \mathrm{C}$. The intensity of the Ru peak at $42.4^{\circ}$ increases gradually with an increase in temperature.

It can therefore be concluded that the Co\Pt bilayers show fcc (111) texture as they are annealed at $300^{\circ} \mathrm{C}$, without a significant presence of the hcp Co phase or $\mathrm{L} 1_{0}$ ordering. The latter only appears at higher temperatures, with the onset of $\mathrm{L} 1_{0}$ ordering taking place above $300^{\circ} \mathrm{C}$. In essence, Equation (1) computes the XRD peak position using the average interplanar distance, which can result from both a coherent epitaxial interface (the expression was derived for superlattices $^{36}$ ) or interdiffusion. To investigate the interdiffusion between $\mathrm{Co}$ and $\mathrm{Pt}$ at the interface, Transmission Electron Microscopy (TEM) and X-Ray Reflectivity (XRR) experiments are performed.

The XRR measurements for samples subjected to $250^{\circ} \mathrm{C}$ and $350^{\circ} \mathrm{C}$ annealing treatments are shown together with fits in Figure 2(a). An illustration of the sensitivity of the fit to the intermixing parameter $\sigma$ at the $\mathrm{Co} / \mathrm{Pt}$ interface is shown in Figure 2(b). $\sigma$ is not sensitive to the $\mathrm{HfO}_{2} \backslash \mathrm{Co}$ interface since the density of both materials is very similar. Clearly, a significant change in XRR characteristics is present 

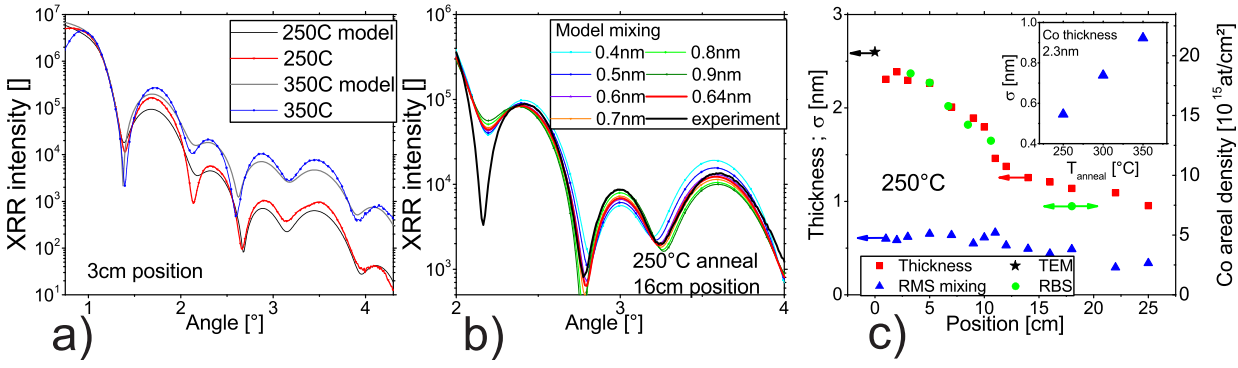

C)
FIG. 2. (a) Experimental and modelled XRR curves for samples annealed at temperatures between $250{ }^{\circ} \mathrm{C}$ and $350^{\circ} \mathrm{C}$. (b) Modeled XRR ratio curves for different rms intermixing values. (c) Thickness and rms intermixing $(\sigma)$ in function of samples position. The inset shows the evolution of rms intermixing in function of the annealing temperature. depending on $\sigma$, which allows for the assessment of intermixing. The resulting fitted thickness of the Co layer as well as the intermixing parameter $\sigma$ are shown in Figure 2(c). $\sigma$ is found to be independent of Co layer thickness and increases strongly with temperature as expected for interdiffusion. Both surface roughness and interdiffusion contribute to $\sigma$ as determined by XRR. Given that the RMS roughness as determined by AFM $(\leq 0.3 \mathrm{~nm})$ is significantly lower than the $\sigma$ values fitted by $\mathrm{XRR}$, the increasing presence of interdiffusion between Co and $\mathrm{Pt}$ in the $250-350^{\circ} \mathrm{C}$ can be inferred from the XRR characteristics. The Co thickness data as calculated from Rutherford Backscattering Spectroscopy (RBS) measurements (assuming fcc Co (111) texture, yielding a linear relationship between the raw RBS data in atoms $/ \mathrm{cm}^{2}$ and the calculated Co thickness) correlate with XRR thickness and are also shown in Figure 2(c). Thicknesses reported in this manuscript are based on the linear interpolation of measured RBS thicknesses.

The AFM measurements (not shown) on as deposited samples show a roughness of $0.17 \mathrm{~nm}$, which increases with the annealing temperature: $0.18 \mathrm{~nm}, 0.19 \mathrm{~nm}$, and $0.31 \mathrm{~nm}$, respectively, for $250{ }^{\circ} \mathrm{C}, 300^{\circ} \mathrm{C}$, and $350{ }^{\circ} \mathrm{C}$ (with an error of $0.01 \mathrm{~nm})$. A strong increase is seen above $300{ }^{\circ} \mathrm{C}$, which coincides with the onset of the upward shift of the central peak in the XRD diffractograms (see Figures 1(a) and 1(b)).

Energy-Dispersive X-Ray Spectroscopy (EDS) is used for probing the distribution of $\mathrm{Si}, \mathrm{C}, \mathrm{Pt}, \mathrm{O}, \mathrm{Hf}, \mathrm{Co}$, and $\mathrm{Ru}$ in function of the depth in the sample. In the depth profile of the as-deposited sample (see Figure 3(a)), the different layers are well distinguished, with Gaussian profiles for the atomic concentrations. The concentration profiles after annealing (see Figure 3(b)) show that the Co and Pt layers have significantly interdiffused, leading in fact to two CoPt alloyed

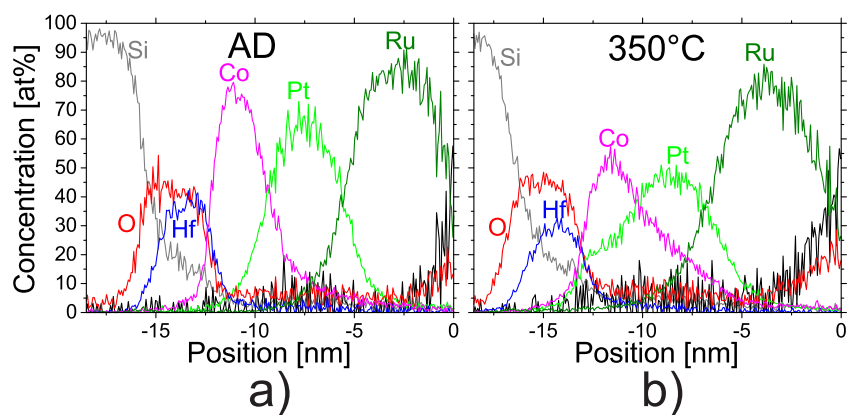

FIG. 3. EDS mapping of the surface layers. The curves show the atomic concentration profiles for $\mathrm{Si}, \mathrm{C}, \mathrm{Pt}, \mathrm{O}, \mathrm{Hf}, \mathrm{Co}$, and Ru. (a) EDS spectrum of an as-deposited multilayer structure with relatively thick Co $(\sim 3.2 \mathrm{~nm})$. (b) EDS spectrum of a similar structure, after annealing at $350^{\circ} \mathrm{C}$ for $10 \mathrm{~min}$. In both figures, the zero of the position axis indicates the approximate position of the surface. layers, one Co rich and one $\mathrm{Pt}$ rich. The $\mathrm{Ru}$ has not significantly diffused. The apparent difference in Hf signals between panels (a) and (b) is due to variations in the oxygen signal, which is not quantitative in EDS. Finally, some Si is present in the $\mathrm{HfO}_{2}$ layer, likely under the form of a layer of silicate. The interfacial silicate is thought to improve the reliability of the oxide, as well as its interfacial quality. ${ }^{37}$

The TEM micrographs (taken at the position shown in Figure 2(c)) are used to correlate the crystallinity information from XRD with the atomic concentrations derived from EDS. The as-deposited sample, Figure 4(a), shows a clear polycrystalline layer of Co. The grains are randomly oriented, with sizes in the order of the thickness of the layer. Little epitaxial ordering was found in the as deposited samples. After the sample is annealed at $350{ }^{\circ} \mathrm{C}$, the $\mathrm{Pt}$ and the Co interdiffused bilayers and the interface between Co and Pt become epitaxial. A layer with slightly darker contrast and the thickness of $\sim 0.6 \mathrm{~nm}$ appears at the interface between $\mathrm{HfO}_{2}$ and the Co-rich Co-Pt layer.

The structural characterization has shown that upon annealing the $\mathrm{HfO}_{2} \backslash \mathrm{Co} \backslash \mathrm{Pt}$ trilayers, the interface between Co and $\mathrm{Pt}$ undergoes epitaxial reordering and interdiffusion.

\section{B. Magnetic characterization}

The magnetic properties of the thin films are investigated with the polar Magneto-Optical Kerr Effect (pMOKE) and Vibrating Sample Magnetometry (VSM).

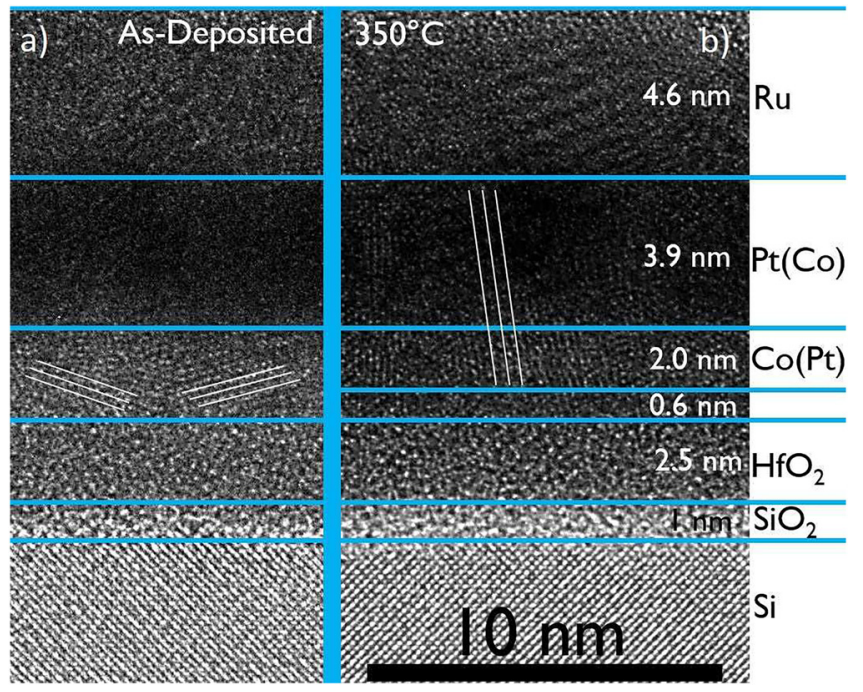

FIG. 4. TEM micrographs of the as-deposited (a) and annealed (b) materials stacks. The thicknesses of the respective layers are indicated on the righthand side of the figure. The crystalline orientation of two adjacent Co grains is indicated with thin white lines. 
The pMOKE measurements of the annealing temperature dependence of the out-of-plane magnetization component vs field for the Co thicknesses of $0.93 \mathrm{~nm}$ (a) and (b) and $1.23 \mathrm{~nm}$ (c) and (d), as measured with Rutherford Backscattering Spectroscopy, are shown in Figure 5. For both thicknesses, the as-deposited thin films show IP magnetic anisotropy. The thin Co layer $\left(\mathrm{t}_{C o}=0.93 \mathrm{~nm}\right)$ features significant PMA from annealing temperatures of $250{ }^{\circ} \mathrm{C}$ and up. The thicker layer $\left(\mathrm{t}_{C o}=1.23 \mathrm{~nm}\right)$ requires a higher annealing temperature of $300^{\circ} \mathrm{C}$ to feature PMA. As $\mathrm{T}_{\text {anneal }}$ is increased to $350^{\circ} \mathrm{C}$, the PMA decreases, similarly to the decrease of $\mathrm{K}_{\text {eff }}$ (not shown). Both the decrease of $\mathrm{K}_{\text {eff }}^{11-13}$ and PMA ${ }^{10}$ in function of the annealing temperature have been reported in the literature. The decrease of PMA and $\mathrm{K}_{\text {eff }}$ can be attributed to the onset of $\mathrm{L} 1_{0}$ ordering, which takes place at $300{ }^{\circ} \mathrm{C}$ (see discussion of XRD results, Figure 1) as well as to significant interdiffusion. ${ }^{6,7,38}$ In Ref. 7, the authors claim that the top Co\Pt interface of a Pt $\backslash \mathrm{Co} \backslash \mathrm{Pt}$ trilayer does not contribute to PMA, mainly due to interdiffusion. Here, we show that there is strong PMA in the Co\Pt bilayers deposited on amorphous $\mathrm{HfO}_{2}$.

Nistor et al. ${ }^{8}$ report on $\mathrm{SiO}_{2} \backslash \mathrm{Co} \backslash \mathrm{Pt}$ trilayers and conclude that annealing can modify the magnetic anisotropy of Pt capped Co thin films deposited on an amorphous template from in-plane to perpendicular. The $\mathrm{HfO}_{2} \backslash \mathrm{CoPt}$ trilayers reported here show the same annealing behaviour (although for lower annealing temperatures), which can similarly be ascribed to the amorphous nature of $\mathrm{HfO}_{2}$. As the temperature is increased further, both the PMA and the $\mathrm{K}_{\text {eff }}$ start to degrade. The MA therefore can be optimized as a function of the annealing temperature. Additionally, the required annealing temperature for featuring PMA increases with increasing Co layer thickness, likely due to the stronger tendency of thicker layers to have an in-plane easy axis. Based on the results shown in Figure 5, an optimal annealing temperature of $300^{\circ} \mathrm{C}$ is chosen for the VSM investigation.

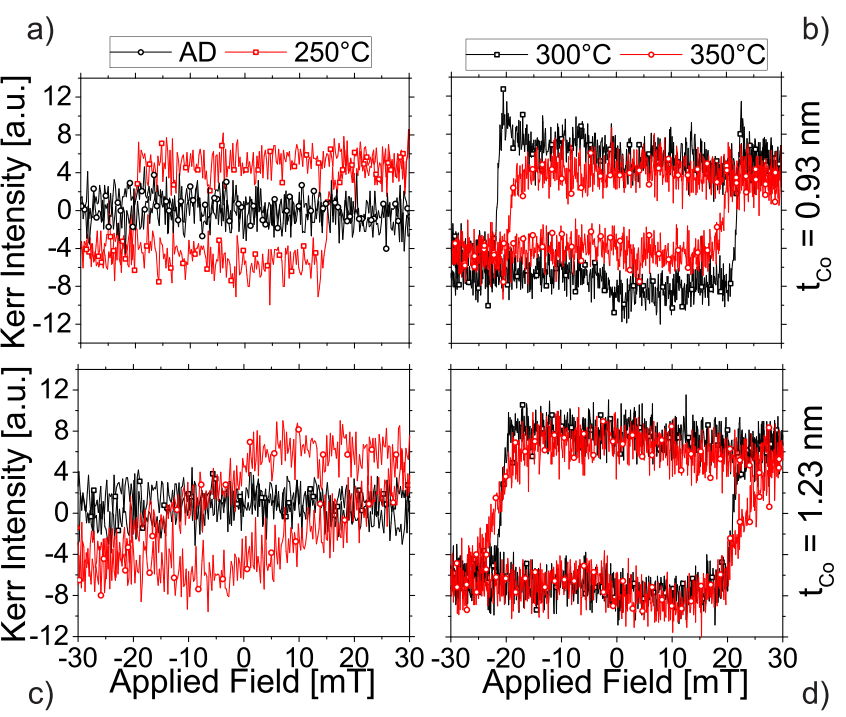

FIG. 5. MOKE perpendicular magnetization curves in function of the annealing temperature for Co thicknesses of $0.93 \mathrm{~nm}$ (a) and (b) and $1.23 \mathrm{~nm}$ (c) and (d).
The magnetic anisotropy energies for different Co thicknesses for samples annealed at $300^{\circ} \mathrm{C}$ are calculated using the following formula making use of the hard axis magnetization curves as measured with VSM:

$$
K_{e f f}=-\mu_{0} \int_{0}^{M_{s, H A}} H d M
$$

For Co thicknesses above $1.55 \mathrm{~nm}$, the effective magnetic anisotropy energy $\mathrm{K}_{\text {eff }}$ decreases linearly with thickness (see Figure 6(a)), and the easy axis lies in-plane. The critical thickness $\mathrm{t}_{c}=1.55 \mathrm{~nm}$ is defined as the Co thickness at which the MA switches from in-plane to perpendicular. Below $t_{c}$, the $\mathrm{K}_{\text {eff }}$ saturates at $0.25 \pm 0.03 \mathrm{~mJ} / \mathrm{m}^{2}$, likely due to the decrease of interfacial anisotropy ascribed to the degradation of the interfaces when the Co thickness decreases. ${ }^{39,40}$ This is comparable to values reported in the literature for similar Co-Pt thin film stacks, ${ }^{3,4}$ which are on the order of $0.3-0.32$ $\mathrm{mJ} / \mathrm{m}^{2}$. An increased annealing temperature has been reported to decrease the $\mathrm{K}_{\text {eff. }}{ }^{11-13}$ The crystallinity of the template can improve the temperature dependence of the $\mathrm{K}_{\text {eff. }}{ }^{11}$ The present results show that it is possible to obtain PMA and a normal $\mathrm{K}_{\text {eff }}$ value at the CoPt interface of significantly interdiffused bilayers deposited onto amorphous $\mathrm{HfO}_{2}$.

By plotting the absolute magnetic moment against the thickness, the presence of a potential magnetic dead layer (MDL) is derived (see Figure 6(b)). The thickness of this dead layer is $0.52 \pm 0.1 \mathrm{~nm}$, which corresponds to the thickness of a darker region seen in the TEM micrograph (see Figure 4) at the interface between $\mathrm{Co}$ and $\mathrm{HfO}_{2}$. The Co layer has two interfaces, one with crystalline Pt and one with amorphous $\mathrm{HfO}_{2}$. The Co\Pt interface is unlikely to display a dead layer. It has good crystallinity, as determined from XRD and TEM (see Figures 1 and 4); Pt tends to be magnetized through magnetic proximity, ${ }^{41}$ and no dead layer has been reported at the $\mathrm{Co} / \mathrm{Pt}$ interface. Local amorphisation $^{42,43}$ or oxidation ${ }^{44}$ of the ferromagnetic layer can lead to loss of magnetic properties. The $\mathrm{HfO}_{2}$ can induce both, so the dead layer is likely located at the $\mathrm{HfO}_{2} \backslash \mathrm{Co}$ interface. HCP Co (0001) deposited on $\mathrm{SiO}_{2}$ is also known to have a magnetic dead layer, with the thickness of about 1 monolayer $(0.4 \mathrm{~nm}) .{ }^{44}$

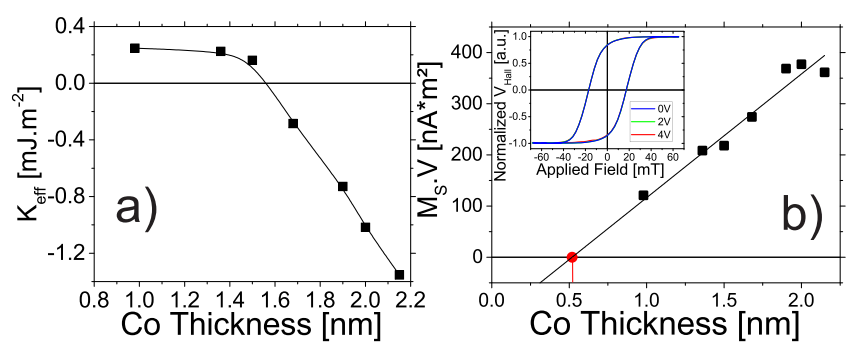

FIG. 6. (a) Effective anisotropy energy in function of Co thickness for samples annealed at $300^{\circ} \mathrm{C}$. (b) Absolute magnetic moment as a function of the Co thickness. A linear fit towards zero magnetic moment allows to determine the thickness of the magnetic dead layer (MDL). The inset shows the Hall voltage in function of the voltage applied across the $\mathrm{SiO}_{2} \mathrm{HfO}_{2}$ dielectric layers. 
To assess whether the MDL influences VCMA at the $\mathrm{HfO}_{2} \backslash \mathrm{Co}$ interface, gated Anomalous Hall Effect (AHE) measurements are used (see inset of Figure 6(b)). The hysteresis loops in the figure are perfectly superimposed, meaning that no VCMA effect is measured for the $\mathrm{HfO}_{2} \backslash \mathrm{Co} \backslash \mathrm{Pt}$ trilayer stacks around $\mathrm{t}_{c}$, despite the high applied electric fields in the order of $10 \mathrm{MV} / \mathrm{m}$. The absence of VCMA is likely due to the presence of the MDL at the $\mathrm{HfO}_{2} \backslash \mathrm{Co}$ interface. As it is the charging of the interface with the dielectric that leads to VCMA,${ }^{16}$ the lack of magnetic properties at that interface likely explains the absence of VCMA. Additionally, Co typically does not show strong VCMA, and the Co\Pt interface, where most of the PMA likely originates is located far away from the charge accumulation.

\section{CONCLUSION}

PMA and VCMA of $\mathrm{HfO}_{2} \backslash \mathrm{CoVt}$ are investigated with the structural and magnetic analysis techniques. An annealing temperature and Co thickness window exist in which PMA can be obtained in $\mathrm{HfO}_{2} \backslash \mathrm{CoVt}$ trilayers. For Co thickness below $1.55 \mathrm{~nm}$ and the annealing temperature between $250^{\circ} \mathrm{C}$ and $300^{\circ} \mathrm{C}$, strong PMA can be obtained with a $\mathrm{K}_{\text {eff }}$ of $0.25 \mathrm{~mJ} / \mathrm{m}^{2}$, comparable to state of the art Co\Pt films. At these annealing temperatures, the Co\Pt interface undergoes significant interdiffusion, as shown by EDS, and simultaneously adopts an epitaxial ordering, as shown with TEM. A VCMA effect proved elusive, which can be attributed to the magnetic dead layer at the $\mathrm{HfO}_{2} \backslash \mathrm{Co}$ interface, but also the weak VCMA effect in Co; the fact that the PMA likely originates in the CoVt interface. In conclusion, we have shown that it is possible to induce PMA in a $\mathrm{HfO}_{2} \backslash \mathrm{CoVPt}$ trilayer and that the appearance of PMA coincides with significant interdiffusion and epitaxial reordering of the Co\Pt interface.

\section{ACKNOWLEDGMENTS}

We wish to acknowledge the support Johan Meersschaut for fruitful discussions on Rutherford Backscattering Spectrometry, Hugo Bender for assisting in the analysis of TEM micrographs and EDS spectra, as well as Sofie Mertens, Yoann Tomczak, and Aaron Thean. We acknowledge the financial support FWO through grant $n$ ZKD0304-00-W01.

${ }^{1}$ M. Johnson, P. Bloemen, F. Den Broeder, and J. De Vries, Rep. Prog. Phys. 59, 1409 (1996).

${ }^{2}$ P. Carcia, J. Appl. Phys. 63, 5066 (1988).

${ }^{3}$ C. Canedy, X. Li, and G. Xiao, Phys. Rev. B 62, 508 (2000).

${ }^{4}$ J. Chatterjee, T. Tahmasebi, S. Mertens, G. S. Kar, T. Min, and J. De Boeck, IEEE Trans. Magn. 50, 1 (2014).

${ }^{5}$ F. Den Broeder, W. Hoving, and P. Bloemen, J. Magn. Magn. Mater. 93, 562 (1991).

${ }^{6}$ G. A. Bertero and R. Sinclair, IEEE Trans. Magn. 31, 3337 (1995).

${ }^{7}$ S. Bandiera, R. Sousa, B. Rodmacq, and B. Dieny, IEEE Magn. Lett. 2, 3000504 (2011).

${ }^{8}$ L. Nistor, B. Rodmacq, S. Auffret, and B. Dieny, Appl. Phys. Lett. 94, 012512 (2009).

${ }^{9}$ H. Yang, M. Chshiev, B. Dieny, J. Lee, A. Manchon, and K. Shin, Phys. Rev. B 84, 054401 (2011).

${ }^{10}$ B. Rodmacq, S. Auffret, B. Dieny, S. Monso, and P. Boyer, J. Appl. Phys. 93, 7513 (2003).
${ }^{11}$ S. Sumi, Y. Kusumoto, Y. Teragaki, K. Torazawa, S. Tsunashima, and S. Uchiyama, J. Appl. Phys. 73, 6835 (1993).

${ }^{12}$ K. Yakushiji, T. Saruya, H. Kubota, A. Fukushima, T. Nagahama, S. Yuasa, and K. Ando, Appl. Phys. Lett. 97, 232508 (2010).

${ }^{13}$ G. Bertero and R. Sinclair, J. Magn. Magn. Mater. 134, 173 (1994).

${ }^{14}$ M. Weisheit, S. Fähler, A. Marty, Y. Souche, C. Poinsignon, and D. Givord, Science 315, 349 (2007).

${ }^{15}$ K. Kita, D. W. Abraham, M. J. Gajek, and D. Worledge, J. Appl. Phys. 112, 033919 (2012).

${ }^{16}$ T. Maruyama, Y. Shiota, T. Nozaki, K. Ohta, N. Toda, M. Mizuguchi, A. Tulapurkar, T. Shinjo, M. Shiraishi, S. Mizukami et al., Nat. Nanotechnol. 4, 158 (2009).

${ }^{17}$ T. Nozaki, Y. Shiota, M. Shiraishi, T. Shinjo, and Y. Suzuki, Appl. Phys. Lett. 96, 022506 (2010).

${ }^{18}$ K. Yamada, H. Kakizakai, K. Shimamura, M. Kawaguchi, S. Fukami, N. Ishiwata, D. Chiba, and T. Ono, Appl. Phys. Express 6, 073004 (2013).

${ }^{19}$ S. Gamble, M. H. Burkhardt, A. Kashuba, R. Allenspach, S. S. Parkin, H. Siegmann, and J. Stöhr, Phys. Rev. Lett. 102, 217201 (2009).

${ }^{20}$ S.-S. Ha, N.-H. Kim, S. Lee, C.-Y. You, Y. Shiota, T. Maruyama, T. Nozaki, and Y. Suzuki, Appl. Phys. Lett. 96, 142512 (2010).

${ }^{21}$ T. Nozaki, K. Yakushiji, S. Tamaru, M. Sekine, R. Matsumoto, M. Konoto, H. Kubota, A. Fukushima, and S. Yuasa, Appl. Phys. Express 6, 073005 (2013).

${ }^{22}$ J. G. Alzate, P. K. Amiri, P. Upadhyaya, S. S. Cherepov, J. Zhu, M. Lewis, R. Dorrance, J. Katine, J. Langer, K. Galatsis et al., IEEE Int. Electron Devices Meet. 2012, 29-35.

${ }^{23}$ C. Fowley, K. Rode, K. Oguz, H. Kurt, and J. Coey, J. Phys. D: Appl. Phys. 44, 305001 (2011).

${ }^{24}$ S. Kanai, M. Endo, S. Ikeda, F. Matsukura, and H. Ohno, J. Phys.: Conf. Ser. 266, 012092 (2011).

${ }^{25}$ H. Meng, R. Sbiaa, M. Akhtar, R. Liu, V. Naik, and C. Wang, Appl. Phys. Lett. 100, 122405 (2012).

${ }^{26}$ U. Bauer, M. Przybylski, J. Kirschner, and G. S. Beach, Nano Lett. 12, 1437 (2012).

${ }^{27}$ Y. Kikuchi, T. Seki, M. Kohda, J. Nitta, and K. Takanashi, J. Phys. D: Appl. Phys. 46, 285002 (2013).

${ }^{28}$ F. Bonell, S. Murakami, Y. Shiota, T. Nozaki, T. Shinjo, and Y. Suzuki, Appl. Phys. Lett. 98, 232510 (2011).

${ }^{29}$ A. Delabie, R. L. Puurunen, B. Brijs, M. Caymax, T. Conard, B. Onsia, O. Richard, W. Vandervorst, C. Zhao, M. M. Heyns et al., J. Appl. Phys. 97, 64104 (2005).

${ }^{30}$ R. W. G. Wyckoff, Crystal Structures 1, 7 (1963).

${ }^{31}$ X. Sun, Z. Jia, Y. Huang, J. Harrell, D. Nikles, K. Sun, and L. Wang, J. Appl. Phys. 95, 6747 (2004)

${ }^{32}$ K. R. Coffey, M. A. Parker, and J. K. Howard, IEEE Trans. Magn. 31, 2737 (1995).

${ }^{33}$ D. Weller, H. Brändle, G. Gorman, C.-J. Lin, and H. Notarys, Appl. Phys. Lett. 61, 2726 (1992).

${ }^{34}$ H. Zeng, M. Yan, N. Powers, and D. J. Sellmyer, Appl. Phys. Lett. 80, 2350 (2002).

${ }^{35}$ D. Treves, J. Jacobs, and E. Sawatzky, J. Appl. Phys. 46, 2760 (1975).

${ }^{36}$ M. R. Khan, C. Chun, G. Felcher, M. Grimsditch, A. Kueny, C. M. Falco, and I. K. Schuller, Phys. Rev. B 27, 7186 (1983).

${ }^{37}$ B. H. Lee, L. Kang, W.-J. Qi, R. Nieh, Y. Jeon, K. Onishi, and J. C. Lee, in IEEE Int. Electron Devices Meet. Tech. Dig. 1999, 133-136.

${ }^{38}$ U. Pustogowa, J. Zabloudil, C. Uiberacker, C. Blaas, P. Weinberger, L. Szunyogh, and C. Sommers, Phys. Rev. B 60, 414 (1999).

${ }^{39}$ M. Kisielewski, A. Maziewski, M. Tekielak, J. Ferré, S. Lemerle, V. Mathet, and C. Chappert, J. Magn. Magn. Mater. 260, 231 (2003).

${ }^{40}$ H. Stillrich, C. Menk, R. Frömter, and H. P. Oepen, J. Magn. Magn. Mater. 322, 1353 (2010).

${ }^{41}$ S.-Y. Huang, X. Fan, D. Qu, Y. Chen, W. Wang, J. Wu, T. Chen, J. Xiao, and C. Chien, Phys. Rev. Lett. 109, 107204 (2012).

${ }^{42}$ K. Oguz, P. Jivrajka, M. Venkatesan, G. Feng, and J. Coey, J. Appl. Phys. 103, 07B526 (2008).

${ }^{43}$ S. Y. Jang, C.-Y. You, S. H. Lim, and S. R. Lee, J. Appl. Phys. 109, 013901 (2011).

${ }^{44}$ S. Entani, M. Kiguchi, S. Ikeda, and K. Saiki, Thin Solid Films 493, 221 (2005). 